\title{
Maintenance Scheduling Optimization for 30kt Heavy Haul Combined Train in Daqin Railway
}

\author{
Yuan Lin ${ }^{1, ~ a, ~ L e i s h a n ~ Z h o u ~}{ }^{1, b}$ and Jinjin Tang ${ }^{1, c^{*}}$ \\ ${ }^{1}$ School of Traffic and Transportation, Beijing Jiaotong University \\ No.3 Shang Yuan Cun, Hai Dian District, Beijing, China.100044 \\ alinyuan@bjtu.edu.cn, ${ }^{\mathrm{b}}$ Ishzhou@bjtu.edu.cn, ${ }^{\mathrm{C} j j t a n g @ b j t u . e d u . c n}$
}

\begin{abstract}
Keywords: railway transportation; maintenance scheduling; modeling and algorithm; Daqin Railway; 30kt heavy haul combined train

Abstract. The freight demand on Daqin Railway is growing yearly, but the maintenance scheduling is almost based on field experience, which makes great impact on transport capacity. Based on maintaining theory, this paper focuses on modeling and algorithm, offering scientific maintenance scheduling for Daqin Railway. With the investigated data, it analyzes the current maintenance scheduling of Daqin Railway. Then the paper models maintenance scheduling based on description of an example illustration, aiming at the least impact of maintenance scheduling on train scheduling. With the algorithm ready, the train operation database is established and the scheduling solving system is developed. Finally, a theoretical maintenance scheduling is provided for 30kt heavy haul combined train in Daqin Railway. The offered maintenance scheduling can meet the actual demand with different maintaining durations.
\end{abstract}

\section{Introduction}

Daqin Railway is known as the important passageway for western coal transportation and the first double-track electrified heavy haul railway in China. It's $653 \mathrm{~km}$ long, stretching from Datong in the west to Qinhuangdao in the east. The freight volume of Daqin Railway is keeping in a steady increasing from 103 million tons in 2002 to 450 million tons in 2014 [1], and it's predicted to reach 460 million tons in 2015. To meet the growing demand of freight transport, the trial of 30kt heavy haul combined train which consists of 5 locomotives and 315 wagons, totally weight $31.5 \mathrm{kt}$, has been carried out successfully on April 2nd, 2014 [2]. As an important part of train scheduling, maintenance scheduling must be scientific and reasonable, which may greatly contribute to improve the transport capacity of Daqin Railway.

Many scholars [3]-[5] have studied on maintenance scheduling in different aspects just with qualitative analysis. Zhen Li [6] quantitatively models the time as well as duration of maintenance scheduling and then designs the algorithm to optimize them, but the line researched is quite simple and the solution is only calculated by manual. Lei Nie [7] models Rectangle-maintenance scheduling to obtain the optimal maintaining time aiming at the minimum of train affected by maintenance scheduling, which has certain reference significance to the heavy-haul railway transportation.

\section{Analysis of Current Maintenance Scheduling}

Currently, Daqin Railway adopts centralized maintenance as well as routine maintenance. Centralized maintenance is adopted continuously during 9:00-12:00 about forty times in spring and autumn yearly. Routine maintenance is adopted once every ten-day during 10:00-12:00 in summer and autumn, while 04:20-06:20 or 5:00-7:00 in winter and spring. Besides, maintenance scheduling are classified into $\mathrm{V}$-maintenance and Rectangle-maintenance according to maintaining type. If we adopt the $\mathrm{V}$-maintenance in electrified railway, the operating track will charge up the maintaining track, leaving great safety risks in operation. So the types of centralized and routine maintenance in Daqin Railway are almost Rectangle-maintenance, which forbids trains to operate on both tracks during maintaining. 
With the data about daily transportation from 2012 to 2014, here makes statistical analysis on average amount of daily loaded trains delivered from Hudong marshalling station in centralized maintenance day, routine maintenance day and regular day in Table 1.

\begin{tabular}{cccc}
\hline Year & $\begin{array}{c}\text { Amount of trains in } \\
\text { centralized maintenance } \\
\text { day }\end{array}$ & $\begin{array}{c}\text { Amount of trains in } \\
\text { routine maintenance } \\
\text { day }\end{array}$ & $\begin{array}{c}\text { Amount of trains in } \\
\text { regular day }\end{array}$ \\
\hline 2012 & 72 & 77 & 87 \\
\hline 2013 & 72 & 79 & 90 \\
\hline 2014 & 71 & 78 & 88 \\
\hline
\end{tabular}

Table 1. Averages of loaded trains delivered in different situations from 2012 to 2014

In 2013, it's shown that the amount of daily loaded trains delivered in centralized maintenance day is 17 less than that in regular day, while the amount in routine maintenance day is 11 less than that in regular day. So the current maintenance scheduling has a great impact on train operation.

The maintaining type in Daqin Railway is almost Rectangle-maintenance, which is of science foundation because of the limit of electrified railway's characteristics. But maintaining time and maintaining duration are only based on field experience without available theory support. What's worse, there is no evaluation mechanism for implementation benefits of the maintenance scheduling. In the near future, Daqin Railway is about to operate the 30kt heavy haul combined train, which sets higher requirement for train scheduling as well as maintenance scheduling. Actually, the current maintenance scheduling should make a great adjustment to adapt to the new requirements. And it's hoped that Daqin Railway can enlarge transport capacity to meet the rising freight demand. Therefore, we must adopt scientific maintenance scheduling, providing powerful guarantee for growth of freight volume. Connected with practice, this paper will model maintenance scheduling based on theory, providing a scientific maintenance scheduling for Daqin Railway.

\section{Modeling Maintenance Scheduling}

Example Illustration. As Fig. 1(a) shows, line $M N$ is a simple railway network with five stations: $M$, $A, B, C, N$, and section $M A, A B, B C, C N$ are maintaining sections divided by power supply sections. Fig. 1(b) is timetable of line $M N$, where we assume section $A B$ adopts a Rectangle-maintenance about one hour at 19:00. The shaded rectangle represents the occupation period and space of maintenance. The trains affected during maintaining are $L_{1}, L_{2}$, and $L_{3}$ in down direction and $L_{4}$ in up direction. 


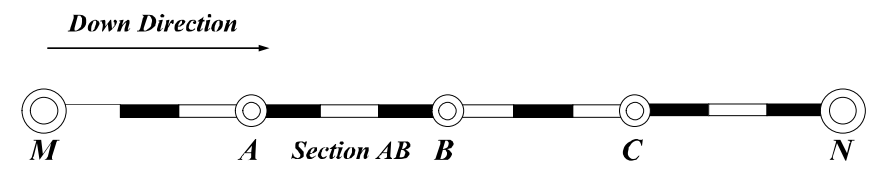

(a) A simple railway network

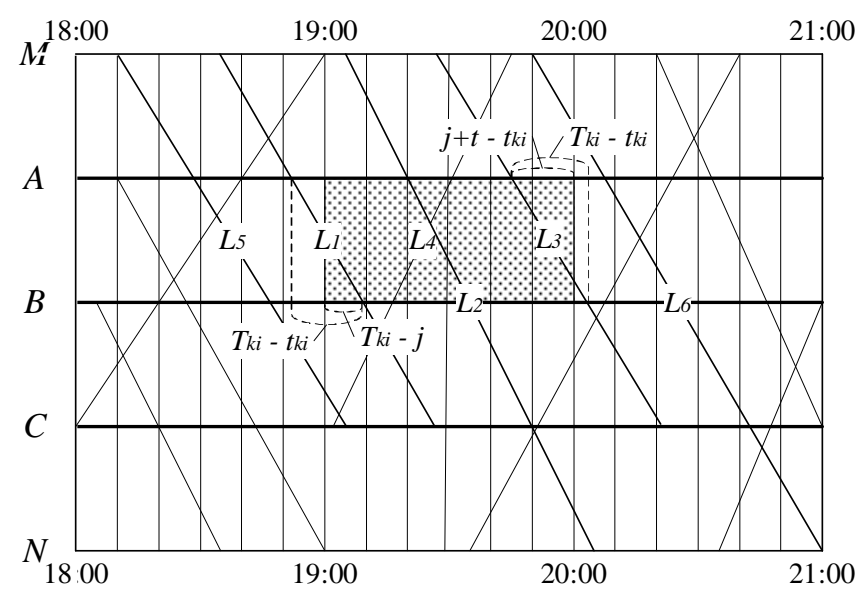

(b) Train timetable of the railway network

Fig. 1. A simple railway network and its train timetable

Parameters Description. Firstly, make explanation to the section and the time.

1) Section: Number the maintaining sections starting from 1 in down direction. For example, $M A$, $A B, B C, C N$ section is numbered as $1,2,3$ and 4 in order. The section is symbolized by $k$.

2) Time: Set 18:00 as number 0 hour and measure the time in minute. It's not to zero if the time is more than $24 \mathrm{~h}$ (1440min). For example, 20:00 is 120min, and 19:00 in next day is $1500 \mathrm{~min}$.

Then, some related parameters are described as follow:

$I$ : Total amount of trains in train timetable.

$i$ : Ordinal number of train in train timetable.

$K$ : Total amount of maintaining sections.

$k$ : Ordinal number of maintaining section start from 1.

$J$ : Departure hour of the latest train.

$j$ : Possible time of starting maintenance and ordinal number of hour starting from 0.

$T$ : Maximum maintaining duration.

$t$ : Maintaining duration.

$t_{k i}$ : Departure time of train $i$ at the former station of section $k$.

$T_{k i}$ : Arrival time of train $i$ at the latter station of section $k$.

$f_{k t j i}$ : Impact coefficient, representing the impact extent on train $i$ when maintenance scheduling is adopted during $[j, j+t]$ in section $k$.

$f_{k t j}$ : Total impact coefficient, representing the impact extent on train scheduling when maintenance scheduling is adopted during $[j, j+t]$ in section $k$.

According to parameters description, if the maintenance scheduling is adopted during $[j, j+t]$ in number $k$ section, the impact coefficient $f_{k t j i}$ of train $k$, is equal to the ratio of $\left[t_{k i}, T_{k i}\right]$ within $[j, j+t]$. 
e.g. $f_{k t j i}=\frac{\left[t_{k i}, T_{k i}\right] \mid[j, j+t]}{\left[t_{k i}, T_{k i}\right]}$

If there are I trains in train timetable, the total impact coefficient is calculated as

$$
f_{k t j}=\sum_{i=1}^{I} f_{k t j i}
$$

Constraints and Objective Function. According to the power supply sections, the line is divided into 22 maintaining sections. So all the researching sections are

$$
k \in\{1,2,3, . .10, \ldots, 21,22\}
$$

Now, the maintenance for Daqin Railway are almost Rectangle-maintenance. To ensure maintaining efficiency, the minimum duration is $2 \mathrm{~h}$ because of subsidiary time for the large machine. In addition, large mechanical efficiency in 5 hours' maintenance is higher than 4 hours' maintenance [6]. In the future, Daqin Railway may elongate maintaining duration to improve its efficiency. Therefore, the maximum duration, $\mathrm{T}$, is determined as $5 \mathrm{~h}$. So the constraint of duration is

$$
t \in\{2,3,4,5\}
$$

Maintenance scheduling need to take period, stuff shifts and repast into account. Because of the complex situation, the paper doesn't restrict the maintaining time, only to fetch the optimal maintaining time for each section in theory. So the constraint of time is

$$
j \in\{0,1,2, . .11, \ldots, 22,23\}
$$

If the section and the duration are determined, we can figure out $f_{k t j}$ of maintenance scheduling adopted in each time and fetch $\min f_{k t j}$. With analysis above, the model of maintenance scheduling for or 30kt heavy haul combined train in Daqin Railway can be formulated as

$$
\begin{aligned}
\min f_{k t j}=\min \sum_{i=1}^{I} f_{k t j i}=\min \sum_{i=1}^{I} \begin{cases}\left(T_{k i}-j\right) /\left(T_{k i}-t_{k i}\right) & t_{k i}<j<T_{k i}<j+t \\
1 & j \leq t_{k i}<T_{k i} \leq j+t \\
\left(j+t-t_{k i}\right) /\left(T_{k i}-t_{k i}\right) & j<t_{k i}<j+t<T_{k i} \\
0 & j>T_{k i} \text { or } j+t<t_{k i}\end{cases} \\
\text { s.t. }\left\{\begin{array}{l}
k \in\{1,2,3, \ldots, 22\} \\
t \in\{2,3,4,5\} \\
j \in\{0,1,2, \ldots, 23\}
\end{array}\right.
\end{aligned}
$$

\section{Model Solution}

Algorithm. To fetch the optimal maintenance scheduling, the model algorithm is designed as follow.

Input: $K$-total amount of maintaining section, $T$-maximum maintaining duration, $J$-departure hour of the latest train and $I$-total amount of train in train timetable.

Output: The optimized maintenance scheduling with four parameters: $k, t, j$ and $\min f_{k t j}$.

Step 1. (Initialization)

$k=1, t=2, j=0, i=1, f_{k t j}=0$; 
Step 2. (Label updating)

For section $k=1$ to $K$

For duration $t=2$ to $T$

For hour $j=0$ to $J$

For train $i=1$ to $I$

If $\left(t_{k i}<j<T_{k i}<j+t\right)$ Then

Update $f_{k t j}=f_{k t j}+\left(T_{k i}-j\right) /\left(T_{k i}-t_{k i}\right)$

End if

If $\left(j \leq t_{k i}<T_{k i} \leq j+t\right)$ Then

Update $f_{k t j}=f_{k t j}+1$

End if

If $\left(j<t_{k i}<j+t<T_{k i}\right)$ Then

Update $f_{k t j}=f_{k t j}+\left(j+t-t_{k i}\right) /\left(T_{k i}-t_{k i}\right)$

End if

If $\left(j>T_{k i}\right.$ or $\left.j+t<t_{k i}\right)$ Then

Update $f_{k t j}=f_{k t j}+0$

End if

Save $k, t, j, f_{k t j}$

End for

End for

End for

End for

Step 3. Fetch and output $\min f_{k t j}$ with the optimal maintenance scheduling.

Train Operation Database. To offer basic data to the algorithm, the train operation database is established based on SQL Server 2013. In the database, there are four column names: $i, k, t_{k i}$ and $T_{k i}$.

Schedule Solving System. This project utilizes C\# module of Visual Studio 2013 to program. After inputting the parameters $K, T, J, I$, the program will run out in 15 seconds. The system's parameters input interface is shown as Fig. 2.

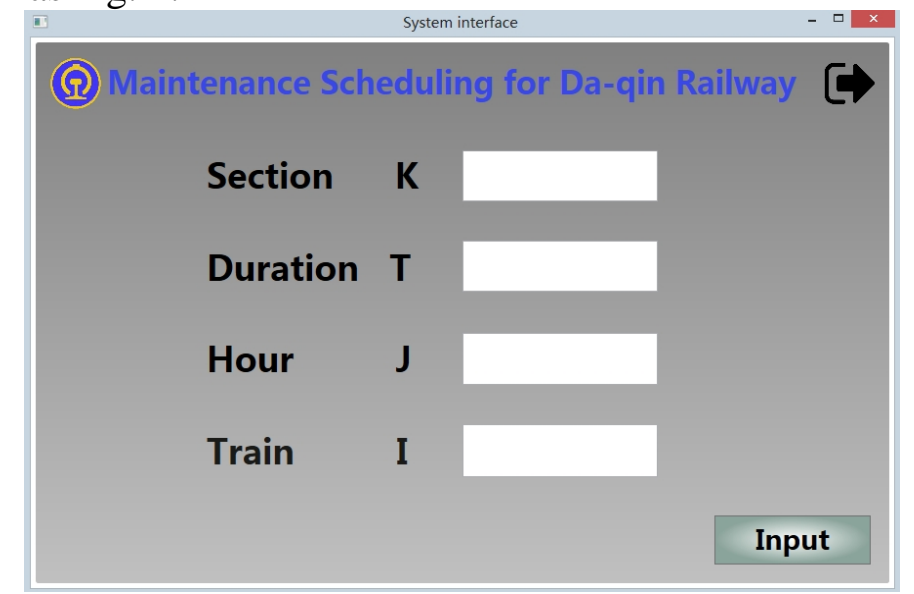

Fig. 2. Parameters input interface

\section{Maintenance Scheduling Optimization for 30kt Heavy Haul Combined Train in Daqin Railway}

Train Scheduling. With the train timetable of 30kt heavy haul combined train in Daqin Railway, we can know that there are 108 loaded trains departing from Hudong marshalling station and 108 empty trains departing from other unloaded stations. So I is 216. The latest train H1960 (Hudong to Chawu) 
departs at No.33 hour, so $J$ is 33 . Then, recording the departure time and arrival time of each train, it finally collects 3678 data in the train operation database.

Solution to Maintenance Scheduling. With the database ready, we can input " $K=22, T=5, J=$ $33, I=216$ " into the textboxes and then run the program. After it runs out, the optimal maintenance scheduling is arranged in Table 2. The table is about maintenance scheduling optimization for each section with different durations.

\begin{tabular}{c|cc|cc|cc|cc}
\hline$t$ & \multicolumn{2}{|c|}{$t=2 h$} & \multicolumn{2}{c|}{$t=3 h$} & \multicolumn{2}{c|}{$t=4 h$} & \multicolumn{2}{c}{$t=5 h$} \\
\hline$k$ & $j$ & $\min f_{k t j}$ & $j$ & $\min f_{k t j}$ & $j$ & $\min f_{k t j}$ & $j$ & $\min f_{k t j}$ \\
\hline 1 & 11 & 11.6 & 23 & 15.4 & 2 & 19.4 & 3 & 24.0 \\
2 & 13 & 11.6 & 23 & 17.3 & 3 & 21.5 & 12 & 26.2 \\
3 & 22 & 14.7 & 18 & 19.5 & 13 & 23.6 & 15 & 28.0 \\
4 & 17 & 15.0 & 13 & 22.5 & 15 & 25.5 & 13 & 30.4 \\
5 & 16 & 15.5 & 6 & 23.4 & 23 & 29.9 & 18 & 34.5 \\
6 & 15 & 13.8 & 15 & 23.8 & 16 & 32.1 & 19 & 38.3 \\
7 & 13 & 13.4 & 20 & 22.6 & 13 & 30.2 & 21 & 39.2 \\
8 & 20 & 14.9 & 13 & 23.8 & 20 & 32.1 & 23 & 39.1 \\
9 & 14 & 14.8 & 13 & 23.2 & 12 & 32.1 & 22 & 38.6 \\
10 & 14 & 15.2 & 14 & 24.1 & 23 & 33.0 & 23 & 39.9 \\
11 & 19 & 14.9 & 22 & 22.7 & 22 & 30.8 & 18 & 37.8 \\
12 & 5 & 15.3 & 13 & 23.1 & 17 & 31.5 & 15 & 38.1 \\
13 & 12 & 12.3 & 11 & 20.2 & 19 & 27.5 & 13 & 32.6 \\
14 & 13 & 12.5 & 12 & 20.4 & 9 & 28.6 & 1 & 33.5 \\
15 & 5 & 11.5 & 20 & 18.7 & 6 & 25.4 & 2 & 31.2 \\
16 & 22 & 12.3 & 12 & 18.9 & 22 & 24.9 & 7 & 29.6 \\
17 & 7 & 10.5 & 21 & 16.3 & 2 & 21.6 & 19 & 26.0 \\
18 & 7 & 9.9 & 2 & 16.3 & 13 & 20.1 & 21 & 25.1 \\
19 & 8 & 3.8 & 7 & 7.1 & 21 & 10.2 & 13 & 13.0 \\
20 & 2 & 4.5 & 17 & 7.2 & 18 & 10.5 & 23 & 12.3 \\
21 & 13 & 1.7 & 13 & 3.8 & 13 & 6.4 & 13 & 7.4 \\
22 & 9 & 2.4 & 9 & 4.4 & 1 & 6.0 & 23 & 7.6 \\
\hline 2
\end{tabular}

Table 2. Maintenance scheduling optimization for 30kt heavy haul combined train

Analysis of Optimizing Maintenance Scheduling. In Table 2, $j$ is the optimal time and $\min f_{k t j}$ is the optimal solution of objective function. Here take $k=6, t=3$ as example. The optimal schedule is $j=15$ and $\min f_{k t j}=23.8$, which means that in section 6 with the duration of $3 \mathrm{~h}$, the optimal time is hour 15 (13:00), and the minimum total impact coefficient is 23.8 .

The optimizing maintenance scheduling for $30 \mathrm{kt}$ heavy haul combined train is adopted for each section with different durations. How to determine the duration depends on the maintaining type and efficiency in practice. The model will figure out the optimizing maintenance scheduling with different durations considering about the actual demand. As for section, the model does not discuss the mutual constraints among different sections, which will lead the solution just to be a local optimum. Thus, it causes deviation between the solution and the reality. For all this, it's still of significance to figure out the optimal maintenance scheduling for single section. In next phase, we will figure out the overall optimization of maintenance scheduling systematically based on the local optimum.

\section{Conclusions}

As an important part of the train scheduling, the maintenance scheduling should avoid being adopted only based on field experience. It is not firstly put forward to aim at maintenance scheduling's least impact on train scheduling, but the existing methods for Daqin Railway are almost by manual calculation and study on the simple track only. In this paper, the schedule solving system could offer 
the optimizing maintenance scheduling with different durations, which actually meets the demand of adjusting the maintaining duration based on efficiency. Furthermore, the paper will take the restrictions of time section, stuff shifts and repast on maintaining time into account. In addition, we hope to put forward the adjustment scheme for the affected trains and establish an evaluation mechanism of implementation benefits for maintenance scheduling.

\section{Acknowledgements}

In this paper, the data is offered by Taiyuan Railway Administration, and the research is supported by China Railway Corporation under grant no.2014X001-B "Transportation Scheduling Optimization for 30kt Combined Train on Daqin Railway".

\section{References}

[1] Daqin Railway Co., Ltd. The Annual Report of Daqin Railway Co., Ltd., Taiyuan, China, 2002-2014.( In Chinese)

[2] Zhiqiang Wang, Study on Security Risk Assessment of 30kt Heavy Haul Combined Train on Daqin Railway, Journal of Science and Technology \&Innovation, pp.122-123, 2014(16). (In Chinese)

[3] Yu Wei, Analysis of Occupation Time of Train Work Diagram by Vertical Window, China Railway Science, vol.25, pp.126-129, 2004. (In Chinese)

[4] Huixiiang Niu, Research on Transportation Organization Mode for 400 Million Ton of Da-Qin Railway, Railway Standard Design, iss.z1, pp.215-217, 2006. (In Chinese)

[5] Feng Chai. Research on All-Around Maintenance Scheduling for Daqin Railway, Degree Thesis of Southwest Jiaotong University, Chengdu, China, 2011. (In Chinese)

[6] Zhen Li, Optimization of All-Around Maintenance Scheduling for Baoshen Railway, Degree Thesis of Beijing Jiaotong University, Beijing, China, 2013. (In Chinese)

[7] Lei Nie, Interaction Analysis between Night Train Operation and Maintenance Time on Passenger Dedicated Railway Line, Journal of Transportation System Engineering and Information Technology, Beijing, China, vol.10, pp.66-72, 2010. (In Chinese) 